\title{
Las fronteras de la Arcadia: naturaleza y estado natural
}

\section{Strosetzki, Christoph}

First published in:

Blecua, Alberto; Arellano, Ignacio; Serés, Guillermo (Hrsg.): El teatro del Siglo de Oro : edición y interpretación. Frankfurt am Main : Vervuert, 2009, S. 441-454

ISBN: 978-3-86527-477-9

(C) 2009 Vervuert Verlag, Frankfurt am Main 


\section{Christoph Strosetzki \\ Universidad de Münster}

La Arcadia se sitúa en el extremo opuesto de la corte y la ciudad. ¿Reside, por tanto, la Arcadia allí donde la naturaleza se encuentra en su estado original o debe, más bien, establecerse una distinción entre esta y el estado natural o presocial? La primera parte de este estudio abordará la cuestión a partir de Eco y Narciso de Calderón mientras que la segunda se dedicará a algunas ideas de la época sobre la naturaleza y el estado natural, debidamente contextualizadas para obtener, con ello, en definitiva, una relación entre las ideas filosóficas y las literarias.

La solidaridad y la sociabilidad caracterizan el mundo pastoril de la pieza teatral de Calderón Eco y Narciso, representada en Madrid en 1661. Los pastores Silvio y Febo veneran a la joven Eco quien, a su vez, sólo tiene ojos para Narciso. Éste había sido criado por su madre en el bosque alejado de toda comunidad humana al haberle sido vaticinado que una voz y una belleza supondrían su fin. Por ello, en repetidas ocasiones rechaza los sentimientos de Eco quien, por ello, y por culpa de un veneno, sólo puede repetir las últimas sílabas de las palabras que otra persona haya pronunciado. Pierde así la facultad de la comunicación humana después de haber abandonado la comunidad 
pastoril y de haberse dirigido al espacio salvaje de Narciso. Evidentemente, tras la bella naturaleza que rodea al mundo pastoril, como si de unos bastidores se tratara, hay un espacio salvaje que es peligroso y perjudicial, puesto que ahí la vida no es social, ni civilizada, ni ordenada; buena muestra de ello dan los siguientes ejemplos: entre los pastores predomina un sentimiento de comunidad; lo que hacen, lo hacen juntos. Si se trata de buscar a alguien, Eco exige: "Todos habemos de ir / juntos»'. Frente a ello, Narciso y su madre, Liríope, viven alejados "destos valles» (v. 135) y se encuentran con frecuencia separados el uno del otro. Liríope se califica a sí misma como "ignorada fiera / destos montes" (vv. 366-367), mientras que por el resto queda caracterizada como "el prodigio a quien / toda esta comarca tiembla" (vv. 377-378) o como "extraño monstruo" (v. 386) y llena de "extrañeza» (v. 382). Al plantearle el pastor Silvio la cuestión de "de qué especie diferente / eres?» (v. 663), parece no pertenecer ya, de manera evidente, al género humano. El salvajismo y el aislamiento de Narciso contrastan así con el mundo pastoril y la comunidad de Eco.

Eco, apreciada y estimada en la comunidad de los pastores, desea convencer a Narciso de que su mundo es el mejor: «¿Cómo te parece el valle? / ¿No es más ameno este sitio / que el monte donde naciste?" (vv. 1583-1585). Los pastores se profesan un aprecio recíproco. Para Bato, Febo es "el pastor / más discreto y entendido / que tiene toda la Arcadia» (vv. 1506-1507), Silvio aparece como "el pastor / más galán» (vv. 1519-1520) y Eco como «la más bella / zagala que el sol ha visto" (vv. 1561-1562). Se justifica, así, que Eco declare al principio que se entregará a todo aquel que sea especialmente galante para con ella (vv. 615-616). Cuando, por el contrario, los pastores hablan de Narciso y Liríope, se trata de un encuentro de "Una hija suya salvaje, / Con un salvajito nieto" (vv. 1305-1306) o surgen lamentos como "Tú no sabes [...] / lo que es tratar con salvajes" (vv. 13101311). Liríope lamenta su desgracia: "Que sólo para ser monstruo / de la fortuna nací" (vv. 673-674). En los encuentros con otras personas Liríope recurre a la violencia y exclama "Mil pedazos te haré antes / que segunda vez me venzas» (vv. 409-410). Mientras que la comunidad pastoril está bien ordenada y se distingue por el trato

' Ver en lo sucesivo: Calderón de la Barca, Eco y' Narciso. Comedia, ed. Aubrun, vv. $928-929$. 
refinado, Narciso y Liríope viven aislados en un ambiente salvaje y se caracterizan por el temor, la violencia y la falta de educación.

Entretanto, Eco puede compararse con Liríope desde el momento en que ésta le administra el veneno, cuyos efectos se describen de la siguiente forma: «Entorpece la lengua / de tal manera que aquél / a quien se le da incapaz / queda del hablar, porque / de las razones no usa / sin pronunciar ni aprender / sino sólo lo que oye, / y aun eso la última vez» (vv. 2388-2395). Dado que ahora Eco ha perdido la facultad de comunicarse de forma razonable con los otros miembros de la comunidad, debe, inevitablemente, retirarse al aislamiento del entorno salvaje: "Huyendo de los poblados / a las asperas montañas / iré y, escondida en ellas, / las más cóncavas estancias / viviré triste y confusa» (vv. 2811-2815).

En Eco y Narciso se establece un contraste entre la comunidad pastoril de Eco con la soledad de Narciso, que vive como un salvaje en el bosque. La comunidad pastoril se ve caracterizada, en este sentido, por conversaciones y modales refinados, mientras que Liríope y Narciso muestran violencia e incapacidad para la comunicación. Si el mundo pastoril recibe una valoración positiva, lo salvaje del bosque, por el contrario, se rechaza y el contacto con los moradores en este estado natural se presenta como el traspaso de una frontera peligrosa.

A continuación, y siguiendo el esquema anunciado en la introducción, daremos un repaso a la historia del estado natural presocial sin pretender con ello polemizar con la exhaustiva y compleja monografía de Fausta Antonucci, El salvaje en la comedia del Siglo de Oro. Historia de un tema de Lope a Calderón ${ }^{2}$. Tampoco es nuestra intención rivalizar con los valiosos estudios de Egido y Varey que, asimismo, se orientan más bien a los fenómenos más que a las ideas subyacentes ${ }^{3}$. Estas enriquecedoras perspectivas fenomenológicas tratarán de ser completadas, en lo que sigue, por la historia de las ideas y de la filosofia. La cuestión del estado natural, ya planteada en la Antigüedad occidental, adquiere a mitad del siglo XVII carta de actualidad, con la aportación de Hobbes de nuevos horizontes con su frase antiaristotélica "Homo homini lupus" con la que se abre una nueva dimensión en el tratamiento de la problemática que nos concierne. Se nos plan-

\footnotetext{
${ }^{2}$ Antonucci, 1995.

3 Egido, 1983; Varey, 1982.
} 
tea, en el campo literario, una dicotomía conflictiva entre la idealización del campo en el género pastoril y el horror del primitivo del estado natural. Paradójico, cuando menos, es, pues, en este sentido, que la sociedad cortesana siguiera anhelando la naturaleza de las novelas pastoriles distanciándose, al mismo tiempo, del estado natural.

Las palabras francesas salvage y sauvage y la española salvaje derivan de la latina silva, es decir, selva o bosque, lo que permite extraer la asociación de lo salvaje con el lugar del bosque. La idea del hombre salvaje, guiado más por el instinto que por el intelecto, desconocedor de la cortesía y con un comportamiento tan grosero como su apariencia, era bien conocida en la Edad Media. Así, el Yvain de Chrétien de Troyes, que vaga por el bosque de Brocéliande acompañado por un león, responde, cuando se le pregunta qué se esconde detrás de su monstruosa figura, que él es a pesar de todo un ser humano ${ }^{4}$. El Perceval de Chrétien crece alejado de la vida caballeresca, pues su madre, tal como hiciera Liríope con Narciso, le educa en la soledad del bosque. Sólo cuando se separa de ella llega a la corte del rey Arturo, donde es instruido por Gornemant de Goort en las formas de la vida cortesana. Ya Tomás de Aquino, apoyándose en Aristóteles, había subrayado la necesidad de sociabilidad del hombre y rechazado el homo silvestris ${ }^{5}$.

De la literatura clásica de la Antigüedad cabe citar la Odisea de Homero, en la que los cíclopes y Polifemo poseen características del hombre salvaje. Hércules, cubierto con la piel del león de Nemea y con una fuerza sobrehumana, no parece sino un hombre salvaje. Horacio, asimismo, califica en sus sátiras a los primeros hombres como especialmente combativos, viendo sus avances hacia la civilización en el refinamiento de la técnica armamentística. Mientras que en un principio se golpeaba con los puños y se arañaba con las uñas, más tarde

${ }^{4}$ Ver Bernheimer, 1952, p. 5.

5 "Dico autem bestiales, puta esse hominem, quem dicunt praegnantes recidentem pueros devorare, vel qualiter bestialitate gaudere aiunt quosdam silvestrium circa pontum", S. Thomae Aquinatis, 1949, p. 368; "Puesto que está determinado al hombre vivir en sociedad, porque cuando vive aislado no puede satisfacer las necesidades de la vida, la sociedad de los muchos será tanto más completa cuantas más exigencias de la vida consiga corresponder», Von Aquin, 1971, p. 9; "homo haturaliter est animal politicum vel sociale», Sancti Thomae Aquinatis, De veritate catholicae fidei contra gentiles, p. 372. 
se hizo uso de palos y se inventaron las armas ${ }^{6}$. Al final de la situación bélica encontramos la introducción de la lengua, de la construcción de las ciudades y de la legislación.

El hecho de que el hombre salvaje carezca no sólo de razón, sino también de la facultad del habla, queda documentado por Shakespeare en el personaje del salvaje Calibán en la obra teatral The Tempest, representada por primera vez en $1611^{7}$. Próspero, que llega a una isla desierta, encuentra allí a Calibán y lo pone a su servicio. El Robinson Crusoe de Daniel Defoe, aparecido en Londres en 1719, es una destacada reedición del mismo tema. El protagonista dispone de conocimientos civilizados cuando observa, como náufrago en una isla, el origen y la autenticidad de la naturaleza. Mediante su destreza artesanal, así como por medio de herramientas y objetos que ha logrado salvar del barco naufragado, comienza a cambiarla y contrapone así su civilización a la naturaleza. Los episodios robinsonianos se encuentran ya en el siglo IX en los relatos de Las mil y una noches, concretamente donde se cuenta el Primer viaje de Simbad el marino, en la noche 252; en el siglo xiII en la segunda Aventiure de Gudrun y en el siglo XVI dentro del relato 67 del Heptamerón de la reina Margarita de Navarra ${ }^{8}$.

$\mathrm{El}$ género literario de la novela de formación parte del estado original de la naturaleza humana como tabula rasa y manifiesta cómo este estado natural puede superarse por medio de la formación y la educación. En ese caso, la naturaleza de este estado primitivo no pocas veces tiene bastantes páginas idílicas y arcádicas. En el Criticón de Gracián, Andrenio es criado por animales y, en un principio, descubre la belleza de la naturaleza antes de emprender su viaje alegórico con Critilo, al final del cual ha acaparado abundantes experiencias. Aparecen aquí en primer plano los conocimientos adquiridos y no los logros civilizadores, como ocurría con Robinson Crusoe.

El estado natural ha sido un tema muy discutido no sólo en la literatura sino también en la Teología. En un principio se trató de la cuestión de la existencia o de la posibilidad de un status hominis in puris naturalibus o un status pure naturalis. En las disputas confesionales, Suárez representó en el siglo xVI la posición católica al citar a otros

${ }^{6}$ Horatius Flaccus, 1953, p. 26 (Satiren I, 3, 99-106)

7 Primer acto, escena segunda, vv. 354-364.

${ }^{8}$ Bien, 1971, p. 279. 
filósofos que habrían tomado en consideración un posicionamiento puramente natural (pure naturalis) que, "si bien nunca ha existido en la realidad, puede considerarse en cambio como posible»" En el lado opuesto protestante señala Quenstedt en 1685: "Los escolásticos y papistas añaden un status hominis in puris naturalibus; pero el hombre nunca ha estado en una condición tal, y tampoco pudo estar» ${ }^{10}$. Así pues, ambas posiciones cristianas están de acuerdo en desmentir la existencia de un estado natural, si bien divergen en lo concerniente a su potencialidad que sería imaginable esta si, como hiciera el filósofo alemán Samuel Pufendorf ${ }^{1}$, mediante la Metafísica sustancial se declarara al hombre como sustancia, a la que se añadirían las cualidades morales, culturales y sociales como meros accidentes. Si, en cambio, nos decidiéramos como Aristóteles por la idea de que el estado natural propio del hombre, como zoon politikon, es social, en ese caso, la cultura formaría parte de la naturaleza o sustancia del hombre.

De forma secularizada continúan los ilustrados franceses del siglo XVIII la discusión sobre el estado natural. Para Montesquieu, la naturaleza puede ser lo primitivo obtenido a través de la abstracción de todo lo institucional y lo transformado históricamente: "Pour les [lois de la nature] connaître bien, il faut considérer un homme avant l'établissement des sociétés» ${ }^{12}$. Como en Suárez, el status naturalis no se asume como históricamente real, sino que se entiende como un estado puramente hipotético que, por motivos metódicos, debe ser aceptado. También en otro punto seculariza la Ilustración algunas posiciones teológicas. De forma similar a la escolástica tardía, que entendía el estado natural como completo egocentrismo, señala Rousseau en Émile que: "L'homme naturel est tout pour lui; il est l'unité numérique, l'entier absolu, qui n'a de rapport qu'à lui-même» ${ }^{13}$. De este aislamiento deduce el ilustrado francés que el hombre carece de capacidad de habla y de reflexión en el estado natural. Los hombres naturales

9 Suarez, De gratia, Prol. 4, Cap. I, núm. 2, cit. según Bien, 1971, p. 296.

10 Citado según Bien, 1971, p. 296.

${ }^{11}$ Ver Samuel Freyherr von Pufendorf, Acht Bücher vom Natur und Völkerrecht, Frankfurt, 1711; del mismo autor: De officio hominis et civis, 1712: aquí lib. II, cap. I: «De statu hominum naturali».

12 Montesquieu, De l'Esprit des Lois, p. 98.

${ }^{13}$ Rousseau, Émile ou de l'éducation; p. 9. 
de Rousseau no son sociables pues no necesitan, en absoluto, de la sociedad.

De modo mucho más crítico fue juzgado el estado natural de los hombres por Bodin y Vives en el siglo xvi. Ambos abogan por una superación lo más rápida posible de dicho estado. En su Methodus ad facilem historiarum cognitionem, aparecido en París en 1566, el escéptico Jean Bodin asume un estado primitivo de salvajismo animal en el que los hombres que vivían dispersos por el bosque y el campo sólo obtenían y poseían lo que habían arrebatado por medio de la fuerza. Sólo se avanzaría por medio de los inventos, de las artes y de las ciencias $^{14}$. Juan Luis Vives subraya en De causis corruptarum artium (1531) que el hombre, a pesar de haber sido creado para la sociedad, por causa de su amor propio "es severo y duro frente a los demás, lo que sería motivo de grandes disturbios, pues cada uno acapararía para sí mismo y para su propio provecho tanto como pudiera obtener por medio de su ingenio o de su fuerza corporal» ${ }^{15}$. Para superar esta situación, Vives deja la responsabilidad en manos de la introducción de la justicia, que pone coto a las manos codiciosas y mantiene alejada la injusticia de la convivencia. Se insinúa aquí una posición que desea la superación de un estado natural peligroso, marcado por intereses antagónicos, mediante la introducción de una justicia socialmente garantizada. El representante más destacado de este posicionamiento en el siglo XVII es Hobbes, cuya valoración negativa del estado natural presentaremos con más detalle a continuación.

Entre 1640 y 1651 sufrió Hobbes el exilio en París. Allí entabló amistad con el franciscano Mersenne y con Pierre Gassendi, quienes elogiaron de forma entusiasta la publicación en lengua latina en el año 1642 de un fragmento de su obra principal, Elementorum philosophiae sectio tertia de cive ${ }^{16}$. Mersenne se había dedicado como teólogo al estado natural del hombre y en su comentario del Génesis había redactado un largo capítulo sobre Adán, quien ya habría llevado consigo el germen de las ciencias conocidas reunidas antes del pecado original ${ }^{17}$.

${ }^{14}$ Ver Zöckler, 1879 , pp. 113 y ss.

15 Vives, Über die Gründe des Verfalls der Künste. De causis cormptarnm artium, pp. 553-555.

${ }^{16}$ Ver Tönnies, 1971 , pp. 23 y ss.

${ }^{17}$ Ver Zöckler, 1879, p. 18; ya los padres de la Iglesia, así como más tarde la dogmática escolástica del siglo XVII, habían otorgado al hombre que todavía no había 
Gassendi se apoya en sus escritos éticos en Hobbes y asume su caracterización del estado natural". Hobbes expone "que el estado de los hombres fuera de la sociedad civil (al que me permito denominar estado natural) no es otro que el de la guerra de todos contra todos en la que todo vale. Además, todos los hombres, apremiados por su naturaleza, tienden a escapar de este estado miserable y repugnante tan pronto como reconocen la miseria; esto, en cualquier caso, sólo es posible si se desprenden de su derecho a todo por medio de la aceptación de contratos" ${ }^{19}$.

¿Qué es lo que pone en relación a los hombres entre sí según Hobbes? Es, o bien esta guerra, que en el estado natural emprenden unos contra otros, o bien, los pactos que acuerdan entre ellos en el subsiguiente estado artificial, es decir, estatal. Así pues, Hobbes no asume como Aristóteles una asociación natural, como la de hombre y mujer, padres - hijo o señor - esclavo, sino que cree que, en principio, el hombre estaría por naturaleza incapacitado para la vida en sociedad, y que algunos hombres continuarían así toda su vida por falta de educación. Hobbes critica la idea aristotélica del hombre como zoon politikon, como ser vivo instalado en la sociedad ${ }^{20}$. Según Aristóteles, el objetivo del hombre es la eudaimonia (dicha suprema), que solamente puede alcanzarse en la Polis. Por eso, sigue Aristóteles, el hombre es por su objetivo, es decir, por su naturaleza, un ser social ${ }^{21}$. Frente a él se posiciona Hobbes: si los hombres fueran por naturaleza seres políticos deberían poder por naturaleza, esto es, por nacimiento, cons-

cometido el pecado original, esto es, el hombre en su estado natural, una triple perfección: intelectual, ética y estética. Ver Zöckler, 1879, pp. 11, 24. Sobre la temática en este contexto de progreso y perfección frente a decadencia: Strosetzki, "Fortschritt und Erfindung im Spannungsfeld von dignitas und miseria hominis", ponencia en el Congreso de Hispanistas en Dresde en 2007.

${ }^{18}$ Ver Ludwig, 2005, p. 18.

${ }^{19}$ Hobbes, Vom Menschen. Vom Bürger, pp. 69-70.

${ }^{20}$ De cive así como el comienzo de sus comentarios en los Elements, en De Cive y en el Leviathan. En Aristóteles aparece la idea del zoon politikon siete veces, a saber, en la Politica, en la Ética Nicomaquea y en la Eudémica y en la Historia de los animales. Ver Wolfers, 1991, p. 61.

${ }^{21}$ "Por tanto, está claro que la ciudad es una de las cosas naturales y que el hombre es, por naturaleza, un animal cívicon, sin embargo, quien vive fuera del Estado "sólo respiraría guerra, porque sería incapaz de unirse con nadie, como sucede a las aves de rapiña", Aristóteles, Política (1252a). 
truir una sociedad con reglas adecuadas, estipuladas contractualmente para la vida en común. Según Hobbes no es éste el caso, pues los hombres nacen como niños a los que les falta la comprensión racional del sentido de semejantes contratos. Puesto que la educación es lo que lleva a esta comprensión razonable, el hombre no sería por naturaleza un ser social ${ }^{22}$.

La filosofia del estado de Hobbes comienza con la teoría del estado natural, en el que que no existen ni el estado ni las instituciones estatales, ni tampoco súbditos o soberanos. Los hombres serían comparables a setas que brotan de la tierra sin que unos estén sometidos a otros ${ }^{23}$. No obstante, este estado no se presenta como paraíso o como Edad de Oro sino, por el contrario, como amenaza permanente de una muerte violenta o como estado bélico de todos contra todos en el que la vida es aislada, insignificante, desagradable y bestial. El afán de gloria, la competencia y la escasez de bienes se ocupan de que cada uno sea un lobo para los demás ${ }^{24}$. Para Hobbes, un hecho consumado revelador del estado natural es "que los hombres separados libremente sólo se unen porque a ello les empujan las necesidades comunes o el ansia de gloria» ${ }^{25}$. Los bienes parecen escasos en vista del deseo humano de una apropiación privada ilimitada. Si varios individuos aspiran a una misma cosa, el conflicto sólo puede resolverse por medio de la lucha. La agresión latente y en cualquier momento a punto de estallar del otro origina junto a la guerra abierta otra subyacente. La razón humana se ocupa de que el hambre futura cree ya al hambriento y la amenaza venidera cree al miedoso y desconfiado. Hobbes entiende

22 «Pues si las personas se quisieran unas a otras por naturaleza, es decir, sólo porque son personas, sería inexplicable por qué unos no quieren en la misma medida a otros si todos son personas en la misma medida", Hobbes, Vom Menschen. Vom Bürger; p. 76.

23 "La naturaleza ha proporcionado a cada uno un derecho; esto es, en el estado natural puro o antes de que los hombres se hubieran unido unos con otros por medio de cualesquiera contratos, estaba permitido a cada uno hacer lo que quisiera y contra quien quisiera, tomar en posesión, utilizar y disfrutar de lo que quisiera y pudiera", Hobbes, Vom Menschen. Vom Bürger, pp. 82 y ss.

24 «Fuera del ser estatal gobierna siempre una guerra de todos contra todos. Por ello es evidente que los hombres, mientras no dispongan de un poder público que les atemorice, se encuentran en aquel estado llamado guerra, en con-creto en una guerra de todos contra todos", Hobbes, Leviathan, p. 104.

25 Hobbes, Vom Menschen. Vom Bürger, p. 78. 
por temor "toda previsión de una desgracia venidera. No sólo el temor, sino también la desconfianza, la sospecha, la precaución y la previsión, para no tener que temer a nada, son propias del miedoso» ${ }^{26}$.

Así, dado que el estado natural está unido a la amenaza permanente de una muerte violenta, si bien al hombre le interesa su propia conservación, la primera ley fundamental de la naturaleza prevé, según Hobbes, el fin del estado bélico y el ingreso en un estado de paz. De este modo, la paz es considerada como un valor y las cualidades humanas a su servicio son reconocidas como virtudes. Una segunda ley prevé que cada uno debe renunciar a su derecho sobre todo, siempre que los demás hagan lo propio.

Como sencilla regla general para el control de la desviación de las leyes naturales, "comprensible incluso para los entendimientos más cortos», Hobbes recomienda: "No hagas a otro lo que no quieres que te hagan a ti» ${ }^{27}$. Las reflexiones de Hobbes acerca del estado natural adquieren fama en el segundo tercio del siglo Xvir con lo que es de suponer que éstas eran conocidas también en España.

Resumiendo, podemos constatar que, según Hobbes, el hombre tiene todo el interés en abandonar el estado natural caracterizado por la guerra de todos contra todos, por la inseguridad, el temor, el afán de reconocimiento, la competencia y por la escasez de bienes. Por ello, estará en situación, mediante la educación, de acabar con el estado bélico así como de ingresar en el estado de paz de un orden social, mediante la introducción de un monopolio coercitivo así como la obligación propia por medio de contratos. En este sentido, en el caso de Hobbes, el hombre procurará abandonar el estado natural de aislamiento y convertirse en un zoon politikon. No obstante, a diferencia de Aristóteles, el hombre no es en el caso de Hobbes un ser sociable por naturaleza. Ambos se alejarían, por tanto, de los homines selvatici o homines silvestres que viven en la naturaleza salvaje del bosque y en un estado natural pre-social, de los primeros porque éstos todavía están lejos de alcanzar su objetivo natural y de los segundos porque éstos todavía no han superado el estado bélico.

La relación entre selva y salvaje, que sugiere la relación etimológica entre silva y sauvage, encontró su ilustración en Narciso y Liríope, 
así como en los "hombres salvajes» de Chrétien de Troyes, en Polifemo y los cíclopes de Homero, en los primeros hombres de las sátiras horacianas, en el Calibán de Shakespeare y en el Robinson Crusoe de Defoe.

Parece sensato, dado que el estado natural es precedente al de la formación y la educación, tomar en consideración la novela de formación de Gracián. En este caso, se mostró como deseo central el abandono del estado natural por medio de la formación y el conocimiento y la perfección alcanzada al final como superpuesta al estado inicial. Mientras que en esta novela se finge el estado natural en las alegorías literarias, su posibilidad en la Teología fue, como hemos visto, objeto de controversias confesionales. Sobre ello se edificaron las argumentaciones secularizadas de los ilustrados, de modo que Montesquieu pudo postular metódicamente el status naturalis como estado hipotético y Rousseau pudo caracterizar a los hombres en estado natural como egocéntricos e insociables. La resolución previa fundamental para responder a si el hombre dispone de capacidad social en el estado natural o posteriormente, depende, como es sabido, de si su sociabilidad se sitúa en su ser, es decir, en su sustancia, o en lo adquirido a través de la educación, es decir, en los accidentes. Aristóteles aboga por el primero de los casos, por el segundo lo hará Hobbes como ya lo habían hecho un siglo antes Bodin y Vives. Todos ellos, sin embargo, abogan unánimemente por una superación del estado natural. A la vista del rechazo general que este produce, es comprensible que los pastores arcádicos se distancien de él y no deseen sobrepasar la naturaleza idílica en favor de un estado natural que les devolvería al estado bélico y les privaría del carácter social, cultivado, en definitiva, ideal, de su sociedad.

¿Y dónde están, para concluir, las fronteras de la Arcadia? Se encuentran allí donde empieza el estado natural. La sociedad pastoril de Calderón se caracteriza por costumbres y conversaciones refinadas y modales cultivados. Más allá de sus límites se mueven Liríope y Narciso, en un bosque en el que el estado natural todavía no ha sido superado, donde dominan el aislamiento, el temor y la violencia. Se muestra así que los pastores de la Arcadia se presentan como opuestos no sólo a la corte y a la ciudad, sino también a la naturaleza en estado puro en la medida en que constituyen una construcción específica artificial. 
Prueba de todo ello son las numerosas discusiones correspondientes en la Literatura, la Filosofía y la Teología, desde Homero hasta Calderón, desde Aristóteles hasta Hobbes y de Suárez a Quenstedt sobre el ámbito de lo presocial, lo incivilizado, lo inculto y lo ignorante, algo que los pastores de la Arcadia hacía mucho que habían superado. 


\section{BiBIIOG RAFÍA}

Antonucci, F., El saluaje en la comedia del siglo de oro. Historia de un tema de Lope a Calderón, Pamplona / Toulouse, Eunsa, 1995.

Bernheimer, R., Wild Men in the Middle Ages, Cambridge, Harvard University Press, 1952.

BIEN, G., "Zum Thema des Naturstands im 17. und 18. Jahrhundert", Archiv für Begriffsgeschichte, 15, 1971, pp. 275-298.

Calderón de la Barca, P., Eco y Narciso. Comedia, ed. Ch. V. Aubrun, Paris, Centre de Recherches de l'Institut d'Études Hispaniques, 1963.

EgIDo, A., "El vestido de salvaje en los autos sacramentales de Calderón", en Serta philologica Fernando Lázaro Carreter, ed. E. Alarcos, D. Alonso y M. Alvar, Madrid, Cátedra, 1983, vol. II, pp. 171-186.

Freyherr von Pufendorf, S., De officio hominis et civis, 1712.

- Acht Bücher vom Natur und Völkerrecht, Frankfurt, 1711.

HobBes, Th., Leviathan, ed. H. Klenner, Hamburg, Meiner, 1996.

- Vom Menschen. Vom Bürger, ed. G. Gawlick, Hamburg, Meiner, 1966.

Horatius Flaccus, Q., Sermones et Epistulae, ed. W. Schöne, München, Ernst Heimeran Verlag, 1953.

Ludwig, B., "Lehrjahre in Exil? Zu einigen Wandlungen in Hobbes' Politischer Philosophie», en Der lange Schatten des Leviathan, ed. D. Hüning, Berlin, Duncker \& Humblot, 2005, pp. 11-27.

Montesquieu, De l'Esprit des Lois, ed. R. Derathé, Paris, Garnier, 1973.

Rousseau, Emile ou de l'éducation, ed. F. et P. Richard, Paris, Garnier, 1964. Strosetzki, Ch., "Fortschritt und Erfindung im Spannungsfeld von dignitas und miseria hominis", en prensa.

Thomae Aquinatis, S., De veritate catholicae fidei contra gentiles, libri quatuor, Luxemburg, Brück, 1881.

- In decem libros ethicorum Aristotelis ad Nicomachum expositio, Roma, Marietti, 1949.

- Über die Herrschaft der Fürsten, Stuttgart, Reclam, 1971.

Tönnies, F., Thomas Hobbes. Leben und Lehre, Stuttgart, Bad Cannstatt, Friedrich Frommann Verlag, 1971.

VArey, J. E., "Cavemen in Calderón (and some cavewomen)", en Approaches to the theater of Calderón, ed. F. McGaha, Washington, University Press of America, 1982, pp. 231-247.

Vives, J. L., Über die Gründe des Verfalls der Künste. De causis corruptarum artium, ed. E. Hidalgo-Serna, München, Fink, 1990. 
Wolfers, B., Geschwätzige Philosophie. Thomas Hobbes' Kritik an Aristoteles, Würzburg, Königshausen \& Neumann, 1991.

Zöckler, O., Die Lehre vom Urstand des Menschen, geschichtlich und dogmatischapologetisch, Gütersloh, Bertelsmann, 1879. 